\title{
The Physical Transient Spectrum for a Multi-Photon V-Type Three-Level Atom Interacting with a Squeezed Coherent Field in the Presence of Nonlinearities
}

\author{
Fahmy K. Faramawy ${ }^{1}$, Abd El-Hameed Abd-Riheem Eied ${ }^{2}$ \\ ${ }^{1}$ Mathematics Department, Faculty of Science, Al-Azhar University, Cairo, Egypt \\ ${ }^{2}$ Department of Mathematics, Faculty of Science, Shaqra University, Shaqra, Saudi Arabia \\ E-mail: fkf54@yahoo.com \\ Received May 24, 2011; revised June 22, 2011; accepted July 1, 2011
}

\begin{abstract}
We study the interaction of a multi-photon three-level atom with a single mode field in a cavity, taking explicitly into account the existence of forms of nonlinearities of both the field and the intensity-dependent atom-field coupling. The analytical forms of the emission spectrum is calculated using the dressed states of the system. The effects of photon multiplicities, mean photon number, detuning, Kerr-like medium and the intensity-dependent coupling functional on the emission spectrum are analyzed.
\end{abstract}

Keywords: Emission Spectrum, Nonlinearities, Multi-Photon Process

\section{Introduction}

The spectrum of spontaneous emission of a V-configuration three-level atom, whose two upper levels are coupled by a classical field and their energy spacing is much larger than the spontaneous emission widths has been investigated [1]. It has been shown that the spontaneously generated interference can induce the spectrum to exhibit six peaks and depend on the phase of the classical field. The effects of a broadband squeezed vacuum on three-level atoms at different configurations ( $\Lambda, V$ and $\Xi$ configurations) have also been investigated [2-5]. Further work has also been done to study the resonance fluorescence spectra of three-level atoms interacting with two coherent lasers and two independent squeezed vacuum [3-5]. The fluorescence spectrum for a strongly driven three-level system in which one of the two photon transition is coupled to a finite-bandwidth squeezed vacuum field has been examined [4]. Quantum interference effects in resonance fluorescence and absorption spectra of a V-type three-level atom damped by a broadband squeezed vacuum, studied in [6].

In recent years, there has been tremendous progress in the ability to generate states of the electromagnetic field with manifestly quantum or nonclassical characteristics experimentally [7-9]. Squeezed states of light are nonclassical states for which the fluctuations in one of two quadrature phase amplitudes of the electromagnetic field drop below the level of fluctuations associated with the vacuum state of the field. Squeezed states therefore provide a field which is in some sense quieter than the vacuum state and hence can be employed to improve measurement precision beyond the standard quantum limits.

The goal of this paper is to shed some light on the emission spectrum for a general three-level system. The model we shall consider is consisting of a single V-type three level atom interacting with a multi-photon one mode field in a perfect cavity, including acceptable kinds of nonlinearities of both the field and the intensitydependent atom-field coupling. To reach our goal it is more convenient to use exact expression for the unitary operator $U(t)$ in the frame of the dressed state formalism. This will be considered in Section 2. In Section 3 we employ the analytical results obtained to find an analytical expression for the emission spectrum by using the finite double-Fourier transform of the two-time field correlation function. By a numerical computation, we examine the influence of photon multiplicities, mean photon number, detuning parameters, the functional dependence of the coupling as well as the nonlinearity parameter on the emission spectrum in Section 4. Finally the conclusions are summarized in Section 5.

\section{Formulation of the Problem}

The Hamiltonian of the system in the rotating-wave app- 
roximation is of the form $(\hbar=1)$

$$
\begin{aligned}
H & =H_{0}+H_{i n} \\
H_{0} & =\sum_{j=1}^{3} \omega_{j} \sigma_{j, j}+\Omega \hat{a}^{+} \hat{a}
\end{aligned}
$$

The operators $\hat{a}$ and $\hat{a}^{+}$are the boson operators for the field satisfying $\left[\hat{a}, \hat{a}^{+}\right]=1$. Where $\omega_{1}, \omega_{2}$ and $\omega_{3}$ are the atomic levels energies $\left(\omega_{1}>\omega_{2}>\omega_{3}\right)$ and $\Omega$ is the field frequency, with the detuning parameters $\Delta_{1}$ and $\Delta_{2}$ (as shown in Figure 1) given by

$$
\Delta_{1}=-k \Omega+\left(\omega_{1}-\omega_{3}\right), \Delta_{2}=-k \Omega+\left(\omega_{2}-\omega_{3}\right)
$$

The interaction part of the Hamiltonian in the presence of an arbitrary nonlinear medium, via multi-photon process $k$ can be written as

$$
\begin{aligned}
H_{\text {in }}= & \Re\left(\hat{a}^{\dagger} \hat{a}\right)+\lambda_{1}\left(\sigma_{13} f_{1}\left(\hat{a}^{\dagger} \hat{a}\right) \hat{a}^{k}+\hat{a}^{+k} f_{1}\left(\hat{a}^{\dagger} \hat{a}\right) \sigma_{31}\right) \\
& +\lambda_{2}\left(\sigma_{23} f_{2}\left(\hat{a}^{\dagger} \hat{a}\right) \hat{a}^{k}+\hat{a}^{+k} f_{2}\left(\hat{a}^{\dagger} \hat{a}\right) \sigma_{32}\right) .
\end{aligned}
$$

$\mathfrak{R}\left(\hat{a}^{\dagger} \hat{a}\right)$ and $f\left(\hat{a}^{\dagger} \hat{a}\right)$ are Hermitian operators functions of photon number operators, such that $\lambda_{1} f_{1}\left(\hat{a}^{\dagger} \hat{a}\right)$ and $\lambda_{2} f_{2}\left(\hat{a}^{\dagger} \hat{a}\right)$ represents an arbitrary intensity-dependent atom-field coupling, while $\mathfrak{R}\left(\hat{a}^{\dagger} \hat{a}\right)$ denotes the one-mode field nonlinearity which can model Kerr-like medium nonlinearity as will be discussed later. The operators $\sigma_{i j}$ satisfy the following commutation relations $\left[\sigma_{i j}, \sigma_{k l}\right]=\sigma_{i l} \delta_{j \kappa}-\sigma_{\kappa j} \delta_{i l},\left[\hat{a}, \sigma_{i j}\right]=0$.

The initial state $\left|\Psi(0)_{A F}\right\rangle$ of the combined atom-field system may be written as

$$
\left|\Psi(0)_{A F}\right\rangle=\left|\Psi(0)_{A}\right\rangle \otimes\left|\Psi(0)_{F}\right\rangle
$$

where $\left|\Psi(0)_{A}\right\rangle=|1\rangle\langle 1|$ the initial state of the atom and $\left|\Psi(0)_{F}\right\rangle=|\Theta\rangle\langle\Theta|$ is the initial state of the field. The initial state $|\Theta\rangle=\sum p^{(n)}|n\rangle$ where the probability amplitude $p^{(n)}$ is defined in the usual manner as $p^{n}=\langle n \mid \Theta\rangle$.

The time evolution between the atom and the field is defined by the unitary evolution operator generated by $H$. Thus $U(t)$ is given by:

$$
U(t) \equiv \exp (-i H t) .
$$

This unitary operator $U(t)$ is written as

$$
\begin{aligned}
U(t) & =\sum_{s=0}^{k-1} \exp \left(-i E_{03}^{(s)} t\right)\left|\Phi^{(s)}\right\rangle\left\langle\Phi^{(s)}\right| \\
& +\sum_{n=k}^{\infty} \sum_{j=1}^{3} \exp \left(-i E_{j}^{(n)} t\right)\left|\Psi_{j}^{(n)}\right\rangle\left\langle\Psi_{j}^{(n)}\right|
\end{aligned}
$$

where $j=1,2,3$ and the eigenvalues

$$
E_{03}^{(s)}=\omega_{3}+\Omega s+\Re(s),(s=0,1, \cdots, k-1)
$$

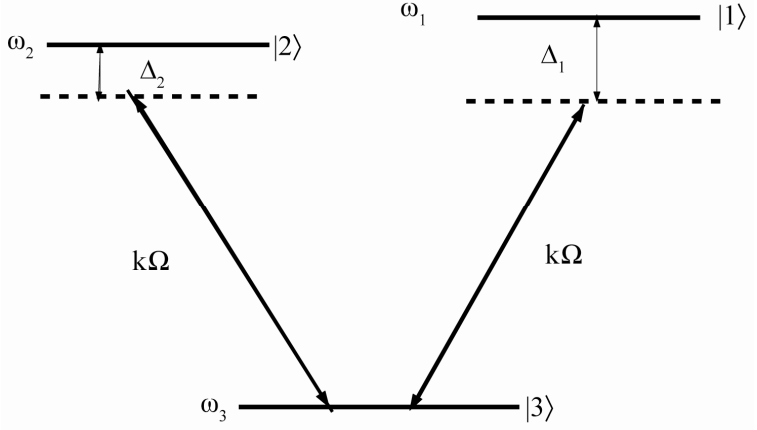

Figure 1. Energy level diagram for a $V$-type three-level atom with $k$-photon detuning $\Delta_{1}, \Delta_{2}$.

$$
E_{j}^{(n)}=-\frac{X_{1}}{3}+\frac{2}{3}\left(\sqrt{X_{1}^{2}-3 X_{2}}\right) \cos \left(\theta_{j}\right)
$$

and

$$
\theta_{j}=\left(\frac{1}{3} \cos ^{-1}\left[\frac{9 X_{1} X_{2}-2 X_{1}^{3}-27 X_{3}}{2\left(X_{1}^{2}-3 X_{2}\right)^{\frac{3}{2}}}\right]+(j-1) \frac{2 \pi}{3}\right),
$$

with

$$
\begin{aligned}
& X_{1}=-\left(r_{1}+r_{2}+r_{3}\right) \\
& X_{2}=-\left[V_{1}^{2}+V_{2}^{2}-r_{1} r_{2}-r_{1} r_{3}-r_{2} r_{3}\right] \\
& X_{3}=r_{2} V_{1}^{2}+r_{1} V_{2}^{2}-r_{1} r_{2} r_{3} \\
& r_{1}=\omega_{1}+\Omega n+\Re(n) \\
& r_{2}=\omega_{2}+\Omega n+\Re(n) \\
& r_{3}=\omega_{3}+\Omega(n+k)+\Re(n+k) \\
& V_{1}=\lambda_{1} f_{1}(n) \sqrt{\frac{(n+k) !}{n !}}, V_{2}=\lambda_{2} f_{2}(n) \sqrt{\frac{(n+k) !}{n !}}
\end{aligned}
$$

and $\left|\Phi^{(s)}\right\rangle,\left|\Psi_{j}^{(n)}\right\rangle$ are the dressed states of the system associated with the eigenvalues $E_{03}^{(s)}$ and $E_{j}^{(n)}, \quad(j=1$, $2,3)$.

$$
\begin{gathered}
\left|\Phi^{(s)}\right\rangle=|s, 3\rangle,(s=0,1, \cdots, k-1) \\
\left|\Psi_{j}^{(n)}\right\rangle=\alpha_{j}^{(n)}|n, 1\rangle+\beta_{j}^{(n)}|n, 2\rangle+\gamma_{j}^{(n)}|n+k, 3\rangle
\end{gathered}
$$

where

$$
\left(\begin{array}{l}
\alpha_{j}^{(n)} \\
\beta_{j}^{(n)} \\
\gamma_{j}^{(n)}
\end{array}\right)=\frac{1}{M}\left(\begin{array}{c}
-V_{1}\left(r_{2}-E_{j}^{(n)}\right) \\
-V_{2}\left(r_{1}-E_{j}^{(n)}\right) \\
\left(r_{1}-E_{j}^{(n)}\right)\left(r_{2}-E_{j}^{(n)}\right)
\end{array}\right)
$$


$M=$

$\sqrt{\left(r_{1}-E_{j}^{(n)}\right)^{2}\left(r_{2}-E_{j}^{(n)}\right)^{2}+V_{1}^{2}\left(r_{2}-E_{j}^{(n)}\right)^{2}+V_{2}^{2}\left(r_{1}-E_{j}^{(n)}\right)^{2}}$

Having obtained the explicit form of the unitary operator $U(t)$, the eigenvalues and the eigenfunctions for the system under consideration, we are therefore in a position to discuss any property related to the atom or the field.

\section{The Physical Transient Spectrum}

In this section we derive the physical transient spectrum $S(v)$ by calculating the Fourier transform of the time averaged dipole-dipole correlation function

$\left\langle\left(\sigma_{13}\left(t_{1}\right)+\sigma_{23}\left(t_{1}\right)\right)\left(\sigma_{31}\left(t_{2}\right)+\sigma_{32}\left(t_{2}\right)\right)\right\rangle$, The physical spectrum $S(v)$ of radiation field emitted by a cavity-bound atom is given by the expression [10].

$$
\begin{aligned}
S(v)= & \Gamma \int_{0}^{T} \mathrm{~d} t_{1} \int_{0}^{T} \mathrm{~d} t_{2} \exp \left[-(\Gamma-i v)\left(T-t_{1}\right)-(\Gamma+i v)\left(T-t_{2}\right)\right] \\
& \times\left\langle\left(\sigma_{13}\left(t_{1}\right)+\sigma_{23}\left(t_{1}\right)\right)\left(\sigma_{31}\left(t_{2}\right)+\sigma_{32}\left(t_{2}\right)\right)\right\rangle .
\end{aligned}
$$

where $T$ is the interaction time and $\Gamma$ is the bandwidth of the filter. After carrying out the various operations we get

$$
\begin{aligned}
& S(v)=\Gamma \sum_{s=0}^{k-1} \sum_{j=1}^{3}\left|p^{(s)}\right|^{2}\left|\alpha_{j}^{(s)}\right|^{2} \Upsilon\left(E_{03}^{(s)}, E_{j}^{(s)}\right) \\
& \times\left[\left|\alpha_{j}^{(s)}\right|^{2}+\alpha_{j}^{*(s)} \beta_{j}^{(s)}+\alpha_{j}^{(s)} \beta_{j}^{*(s)}+\left|\beta_{j}^{(s)}\right|^{2}\right] \\
& +\Gamma \sum_{n=k}^{\infty} \sum_{i=1}^{3} \sum_{j=1}^{3}\left|p^{(n)}\right|^{2}\left|\alpha_{j}^{(n)}\right|^{2}\left|\gamma_{i}^{(n-k)}\right|^{2} \Upsilon\left(E_{i}^{(n-k)}, E_{j}^{(n)}\right) \\
& \times\left[\left|\alpha_{j}^{(n)}\right|^{2}+\alpha_{j}^{*(n)} \beta_{j}^{(n)}+\alpha_{j}^{(n)} \beta_{j}^{*(n)}+\left|\beta_{j}^{(n)}\right|^{2}\right]
\end{aligned}
$$

where

$$
\Upsilon(x, y)=\left[\frac{1+\exp (-2 \Gamma T)-2 \exp (-\Gamma T) \cos (v+x-y) T}{\Gamma^{2}+(v+x-y)^{2}}\right]
$$

Thus the time averaged spectrum consists of resonant structures which arise from transitions among different dressed states. The final structure of the time averaged spectrum will depend on the form of the input photon distribution $p^{(n)}$. Due to the quantum interference between component states the oscillations in the cavity field become composed of different component states.

\section{Results and Discussion}

On the basis of the analytical solution presented in a previous section, we shall study numerically the physical transient spectrum in a a squeezed coherent initial field. The photon number distribution for a squeezed coherent state [11] can be written as

$$
\begin{aligned}
\left|P_{n}\right|^{2}= & s \frac{(\tanh r)^{n}}{2^{n} n ! \cosh r}\left|H_{n}\left(\frac{\varepsilon}{\sqrt{2 \cosh r \sinh r}}\right)\right|^{2} \\
& \times \exp \left[-|\varepsilon|^{2}+\tanh r \operatorname{Re}(\varepsilon)^{2}\right],
\end{aligned}
$$

where, $\varepsilon=\alpha \cosh r+\alpha^{*} \sinh r, \alpha=|\alpha| \exp (i \varsigma)$ and $H_{n}$ is the Hermite polynomial. We suppose here the minor axis of the ellipse, representing the direction of squeezing, parallel to the coordinate of the field oscillator. The initial phase $\varsigma$ of $\alpha$ is the angle between the direction of coherent excitation and the direction of squeezing. The mean photon number of this field is equal to $\bar{n}=|\alpha|^{2}+\sinh ^{2} r$. Putting $r=0$ we get the photon distribution for an initial coherent state with $\bar{n}=|\alpha|^{2}$ whereas for $\alpha=0$ the photon distribution for an initial squeezed vacuum state with $\bar{n}=\sinh ^{2} r$ is recovered. The latter distribution is oscillatory with zeros for odd $n$.

By means of dressed atom states, and quantum beats we know that when the number $n$ changes by $k$ unit, this coupling describes the spontaneous emission of $k$ photon with a frequency close to $\Omega$. Since the dressed state $\left|\Psi_{3}^{(n-k)}\right\rangle$ is totally decoupled from the other dressed states, it can not be populated $[12,13]$. Then the field can only couple such transitions where the final states are either $\left|\Psi_{1}\right\rangle$ or $\left|\Psi_{2}\right\rangle$ but not $\left|\Psi_{3}\right\rangle \quad[12,14]$. So, there are only six possible allowed spontaneous decays from the three perturbed states of $E^{(n)}$ (where $\left.E^{(n)}=\left\{\left|\Psi_{1}^{(n)}\right\rangle,\left|\Psi_{2}^{(n)}\right\rangle,\left|\Psi_{3}^{(n)}\right\rangle\right\}\right)$ to lower multiplicities $E_{j}^{(n-k)}[15,16]$. Generally they are asymmetric, because the intensities of the symmetrically placed sidebands are not equal. While at exact resonance the spectrum becomes symmetric [16]. Due to the non population of the dressed state $\left|\Psi_{3}\right\rangle$ the two transitions $\left|\Psi_{3}\right\rangle \rightarrow\left|\Psi_{1}\right\rangle$ and $\left|\Psi_{3}\right\rangle \rightarrow\left|\Psi_{2}\right\rangle$ vanish [12-14]. Hence, the emission spectrum of $V$-type three-level atom for a single cavity field has four-peak structure. In what follows we shall consider the effect of men photon number, detuning parameters $\Delta_{1}, \Delta_{2}$, intensity dependent coupling $f_{i}(n)$ and the nonlinear Kerr medium parameter $\chi$ on the spectrum of the system under consideration.

\subsection{Effect of Multiplicity and Mean Photon Number}

For $k=1$ small $\bar{n}$ Rabi vacuum peaks dominate the spectrum see Figure 2.1(a). While by increasing the 
mean photon number of the cavity field the effect of the vacuum state diminishes and we note that there is a central deep gap surrounded by two symmetric spikes beside a two symmetric peaks however with structure appear as one moves away from the center as shown in Figure 2.2(a). This is due to that the central two peaks located at $\left(v+E_{1}^{(n-k)}-E_{1}^{(n)}\right)$ and $\left(v+E_{2}^{(n-k)}-E_{2}^{(n)}\right)$ coalescence into each other, giving a single double peak at the center of the spectrum, while the other two peaks giving the symmetric sidebands around the central structure (see Figure 2.2(a)). With further increase in the mean photon number and hence the variance the spectrum not only become quite rich, and the gap depth and width decrease because the central two peaks coalescence into each other further but also, the sidebands move away from the central line, and its height decreases while it gets wider and wider as long as the mean photon number $\bar{n}$ increases. Hance, at sufficiently large mean photon number the central two peaks merge to a single peak leading to a three-peak structure. In Figure 2(b) where $k=2$ the situation is completely changed, we note the appearance of two wall separated central spikes surrounded by two symmetric groups of small spikes, which their heights having a maximum for the small middle spikes. Also, the central structure which observed at $k=1$ disappearing here. The two central spikes which nearest to the center becomes higher and narrower as the mean photon number increase. But for $k=3$ the spectra dived in to two symmetric groups of spikes located around the center. By increasing the values of $\bar{n}$ the spectrum is quite rich. Also, we can observe that the side peaks move away from the central line, and its
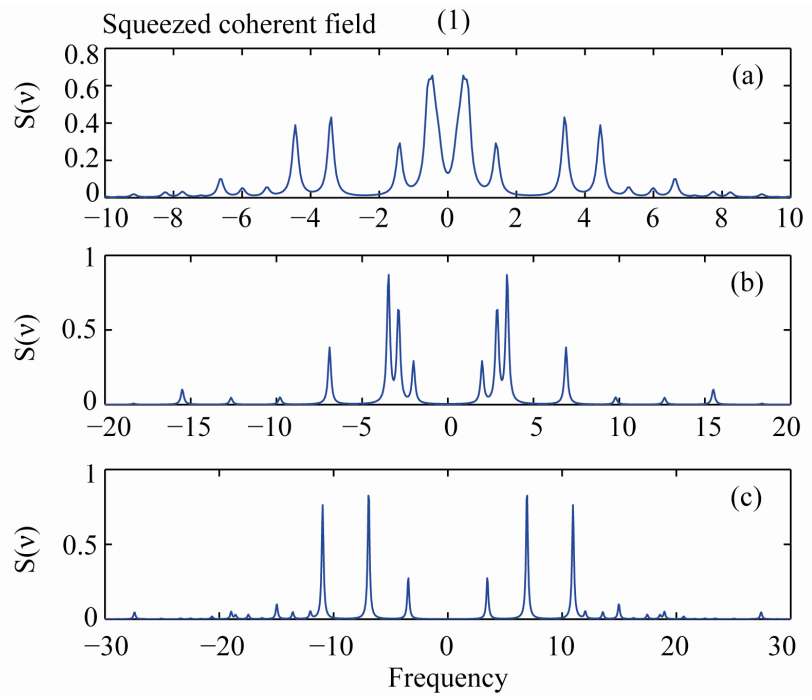

height decrease by increasing the mean photon number see Figure 2.2(c). Finally, as the photon multiplicities number increase, the number of allowed transition between the dressed states increase and hence number of peaks appearing in the spectrum increase as $k$ increase (compare frames in Figures 2.1 and 2.2).

\subsection{Effect of Detuning}

As we compare the figure Figure 3 and Figure 2.2 where the case of absence of detuning is considered, we generally may say that detuning adds asymmetry to the spectrum and the shape of the spectrum is changed on both sides of the central line. The left spikes is suppressed on changing $\Delta_{1}, \Delta_{2}$, while the right spikes is moving away from the resonant frequency and becomes higher as the values of the detuning parameters $\Delta_{1}, \Delta_{2}$ increase (compare frames 1, 2 in Figure 3. Also, the peaks at the left hand side decrease gradually, so that it disappear for a large values of the detuning parameters $\Delta_{1}, \Delta_{2}$ as shown in Figure 3.2. But, these phenomena takes place in a faster way as $\mathrm{k}$ decrease (compare frames a,b,c in Figure 3.2. Finally, not only the number of peaks, there position and there maximum heights depends on the detuning parameters $\Delta_{1}, \Delta_{2}$ but, also the photon number multiplicity $k$.

\subsection{Effect of Kerr Medium}

Now we will turn our attention to the effect on the spectrum $S(v)$ of the nonlinearity of the field with a Kerr-type medium due to the term $\mathfrak{R}(n)$ being taken in
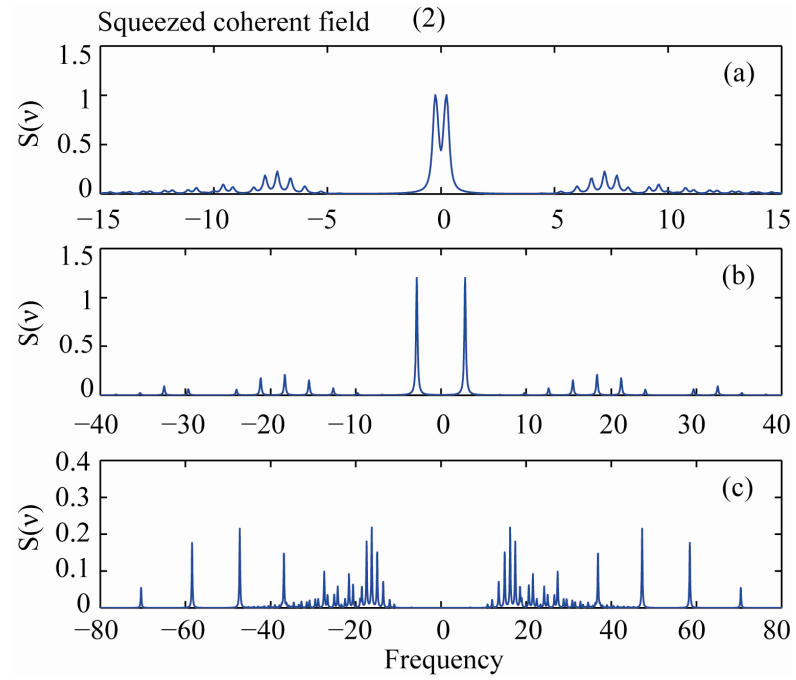

Figure 2. The evolution of the function $S(v)$ in a perfect cavity as a function of $(v-k \Omega) / \sqrt{\lambda_{1} \lambda_{2}} \quad$ with $\quad \lambda_{1,2}=1, \quad \Delta_{1,2}=0$, $\chi=0, \Gamma=0.1, \varsigma=0, f_{1,2}(n)=1, T=100$ and $(a) k=1 ;(b) k=2 ;(c) k=3$ with $(1) r=1, a=1 ;(2) r=1.1, \alpha=\sqrt{5}$. 

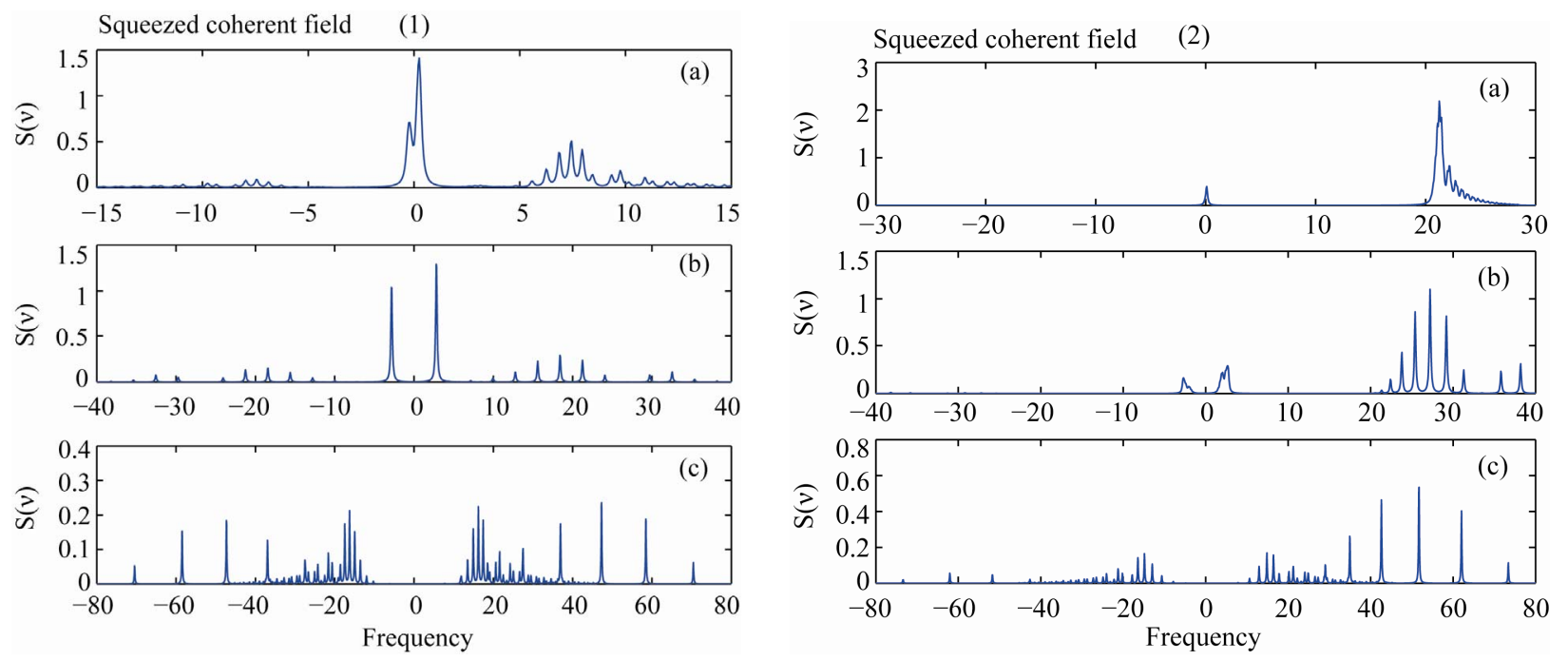

Figure 3. The same as Figure 2.2 but with, (1) $\Delta_{1}=5, \Delta_{2}=4$; (2) $\Delta_{1}=\Delta_{2}=20$.

the form $\chi n(n-1)$, where $\chi$ is related to the thirdorder nonlinear susceptibility. In fact the optical Kerr effect is one of the most extensively studied phenomenon in the field of nonlinear optics because of its applications. The addition of the Kerr-like medium parameter to the problem adds asymmetry to the spectrum that can be seen from comparison of the cases considered in Figure 4 with Figures 2.2. The right side peaks are suppressed while the left side peaks gain height and becomes narrower gradually as the values of $\chi$ increased see Figure 4.2. So that the right side hand peaks nearly disappear for a large values of $\chi$ see Figure 4.2. But this phenomena takes place in a slow way as $\mathrm{k}$ increase. We may conclude that, the effect of the Kerr-like medium is opposite to the effect of detuning. Furthermore, the effect of the nonlinear medium on the spectrum of the emitted light is the shift of the spectrum to the left and changing the amplitudes of the peaks depending on the value of $\chi, k$. The maximum height of peaks increase as $\chi$ increases for all values of $\mathrm{k}$. While the maximum height of peaks decrease as $\mathrm{k}$ increases for all values of $\chi$.

\subsection{Effect of Intensity Dependent Coupling Functional}

In Figure 5 we study the effect of different functionals of intensity dependent coupling $f_{1}(n), f_{2}(n)$ on the emission spectrum $S(v)$. When $f_{1}(n)=f_{2}(n)=\sqrt{n+1}$ the range of the spectrum is extended due to the larger stepwise excitation than that for $f_{1}(n)=f_{2}(n)=1$. Also, the spectra divided into two groups of peaks which are symmetrically located around the central frequency for all values $k$ (see Figure 5.1. Furthermore, not only the maximum height of peaks decrease but, the number of these peaks increases as $k$ increase. When we consider $f_{1}(n)=f_{2}(n)=\frac{1}{\sqrt{n+1}}$ the stepwise excitation becomes smaller than that in the case $f_{1}(n)=f_{2}(n)=1$. So that the sidebands become closer to each other, hence the number of peaks and the range of the spectrum decreases for all values of $k$ see Figure 5.2. For $k=1$ we get a situation similar to a Mollow spectrum (i.e only three peaks appears)which one gets in semiclassical fields see Figure 5.2(a). While for $k=2$ we note a deep gap between two higher wall spikes at the center surrounded by two lower side bands see Figure 5.2(b). For $k=3$ the two wall spikes which nearest to the center gain height (compare Figure 5.2(c) with Figure 2.2(c)). Finally, the spectrum can be controlled by choosing the right intensity-dependent coupling functional.

\section{Conclusions}

We have investigated the emission spectrum for a multiphoton V-type three-level atom, taking into account arbitrary forms of nonlinearities of both the field and the intensity-dependent atom-field coupling. The spectrum is calculated when the field initially in a squeezed coherent state. We have explored the influence of various parameters of the system on the emission spectrum. It is observed that

- For $k=1$ the spectrum of V-type three-level atom for the cavity field has four-peak structure. But for sufficiently large values of the mean photon number the spectrum tends to a three peak structure.

- As the photon multiplicities number increase, the number of allowed transition between the dressed states increase and hence number of peaks increase as $k$ icrease. 

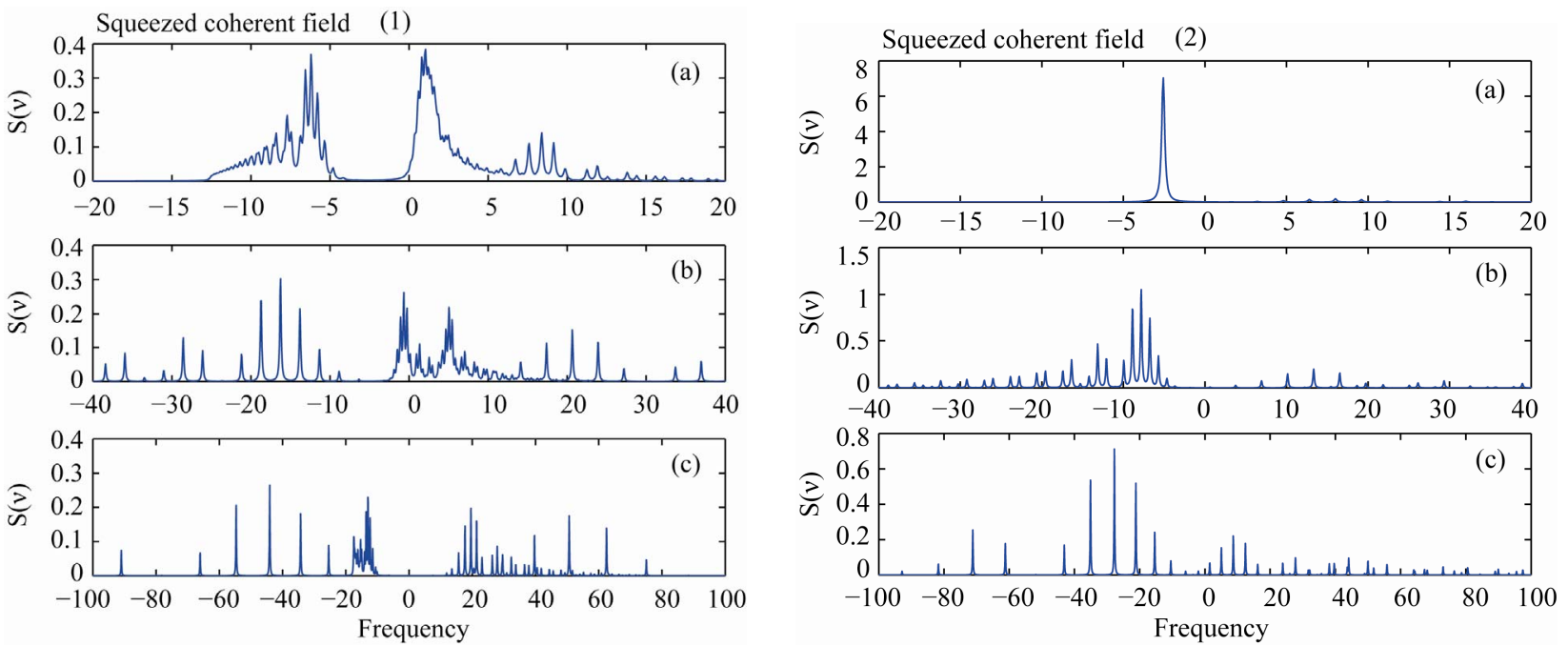

Figure 4. The same as Figure 2.2 but with, (1) $\chi=0.1$; (2) $\chi=0.8$.
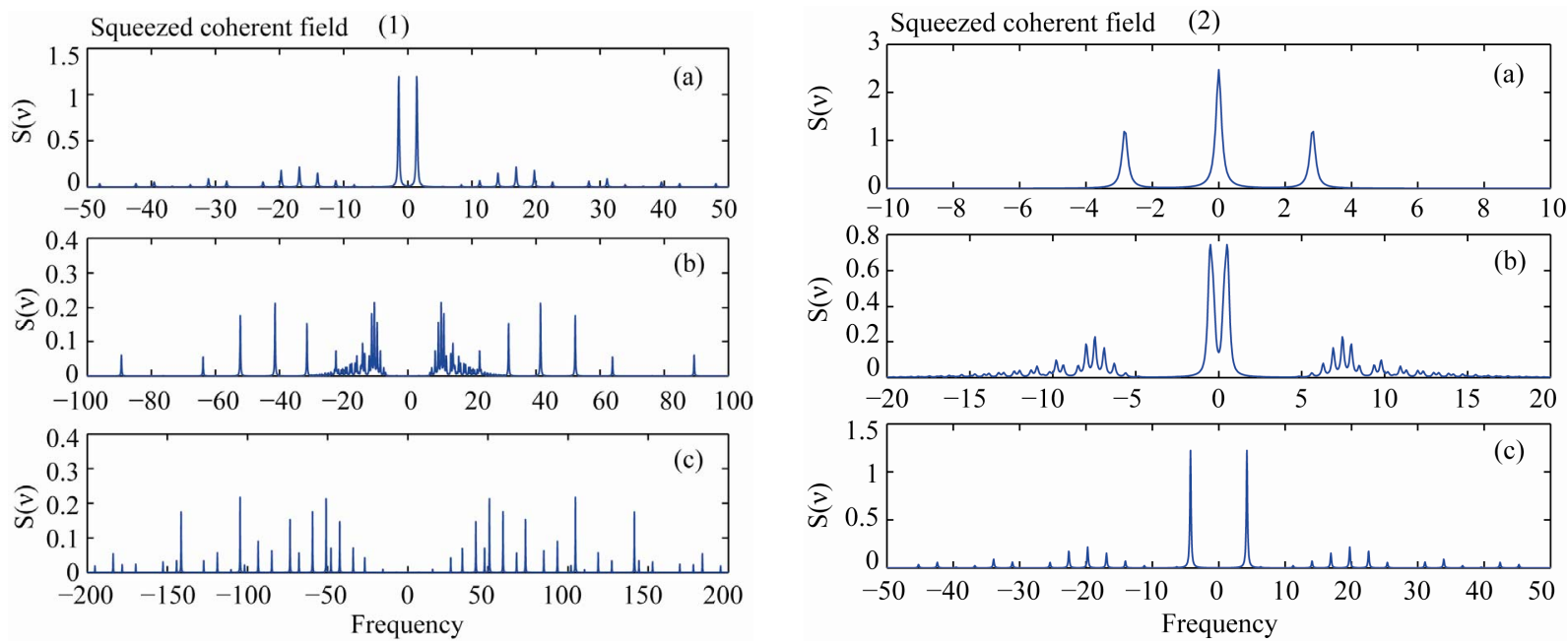

Figure 5. The same as Figure 2.2 but (1) $f_{1}(n)=f_{2}(n)=\sqrt{n} ;(2) \quad f_{1}(n)=f_{2}(n)=\frac{1}{\sqrt{n+1}}$.

- The peak position is associated with not only the photon number $(\bar{n})$ and the photon multiplicity number $k$ but also the intensity-dependent atom-field coupling constant $\lambda_{i} f_{i}(n)$.

- The heights of the spectrum components becomes shorter and the distances between them is larger as the mean photon number increased.

- The symmetry shown in the standard three-level atom model for the spectra is no longer present once Kerr effect or detuning is considered.

- The effect of detuning on the spectrum of the emitted light is twofold. The first effect is the shift of the spectrum to the right side. The second effect is the dependence of the amplitudes and heights of the peaks on $\Delta_{i}$.

- The Kerr medium has an effect opposite to the effect of the detuning, where the earlier has shorter elements. Also, the heights and widths of the peaks not only depend on the photon multiplicity but also on the value of $\chi$. Consequently, changes in the detuning and the Kerr medium parameters can show in the spectra, and hence the heights of the peaks, their shifts and widths are altered compared with the case of resonance.

- The strong field effects can be produced by choosing the right parameters for these nonlinearities.

\section{References}

[1] C. Hooijer, G.-X. Li, K. Allaart and D. Lenstra, "Spontaneous emission in a V-Type Three-Level Atom Driven by a Classical Field," Physics Letters A, Vol. 263, No. 4-6, 1999, pp. 250-256. 


\section{doi:10.1016/S0375-9601(99)00718-5}

[2] Z. Ficek and P. D. Drummond, "Three-Level Atom in a Broadband Squeezed Vacuum Field. I. General Theory," Physical Review A, Vol. 43, No. 11, 1991, pp. 6247-6257. doi:10.1103/PhysRevA.43.6247

[3] S. Smart and S. Swain, "Three-Level Atom in a Squeezed Vacuum II. Resonance Fluorescence," Journal of Modern Optics, Vol. 41, No. 6, 1994, pp. 1055-1077. doi:10.1080/09500349414551021

[4] B. J. Dalton, M. R. Ferguson and Z. Ficek, "Resonance Fluorescence Spectra of Three-Level Atoms in a Squeezed Vacuum," Physical Review A, Vol. 54, No. 3, 1996, pp. 2379-2390. doi:10.1103/PhysRevA.54.2379

[5] B. J. Dalton M. Bostick and Z. Fizec, "Probe Absorption Spectra for Driven Atomic Systems in a Narrow Bandwidth Squeezed Vacuum," Physical Review A, Vol. 53, No. 6, 1996, pp. 4439-4439. doi:10.1103/PhysRevA.53.4439

[6] O. G. Calderon, F. Carreno and M. A. Anton, "Quantum Interference Effects in Resonance Fluorescence and Absorption Spectra of a-Type Three-Level Atom Damped by a Broadband Squeezed Vacuum," Optics Communications, Vol. 221, No. 4-6, 2003, pp. 365-385. doi:10.1016/S0030-4018(03)01490-1

[7] D. F. Walls and G. J. Milburn, "Quantum Optics," Springer-Verlag, Berlin, 1994.

[8] C. M. Caves and B. L. Schumaker, "New Formalism for Two-Photon Quantum Optics. I. Quadrature Phase and Squeezed States," Physical Review A, Vol. 31, No. 5, 1985, pp. 3068-3092. doi:10.1103/PhysRevA.31.3068

[9] V. V. Dodonov, "Nonclassical States in Quantum Optics: A Squeezed Review of the First 75 Years," Journal of
Optics B, Vol. 4, No. 1, 2002, p. R1. doi:10.1088/1464-4266/4/1/201

[10] J. H. Eberly and K. Wodkiewicz, "The Time-Dependent Physical Spectrum of Light," Journal of the Optical Society of America, Vol. 67, No. 9, 1977, pp. 1252-1261. doi:10.1364/JOSA.67.001252

[11] R. Loudon, "Quantum Statistics of Linear and Nonlinear Optical Phenomena," Optica Acta: International Journal of Optics, Vol. 31, No. 8, 1984, pp. 847-852. doi: $10.1080 / 713821593$

[12] S.-Y. Zhu, R. C. F. Chan and C. P. Lee, "Spontaneous Emission from a Three-Level Atom," Physical Review A, Vol. 52, No. 1, 1995, pp. 710-716. doi:10.1103/PhysRevA.52.710

[13] G. X. Li, K. Allaart, C. Hooijer and D. Lenstra, "Spontaneous Emission in a V-Type Three-Level Atom Driven by a Classical Field," Physics Letters A, Vol. 263, No. 4-6, 1999, pp. 250-256. doi:10.1016/S0375-9601(99)00718-5

[14] P. Dong and S. H. Tang, "Absorption Spectrum of a V-Type Three-Level Atom Driven by a Coherent Field," Physical Review A, Vol. 65, 2002, Article ID 033816, 10 Pages.

[15] C. Cohen-Tannoudji and S. Reynaud, "Dressed-Atom Description of Resonance Fluorescence and Absorption Spectra of a Multi-Level Atom in an Intense Laser Beam," Journal of Physics B, Vol. 10, No. 3, 1977, p. 345. doi:10.1088/0022-3700/10/3/005

[16] C. Cohen-Tannoudji and S. Reynaud, "Simultaneous Saturation of Two Atomic Transitions Sharing a Common Level," Journal of Physics B, Vol. 10, No. 12, 1977, p. 2311. doi: $10.1088 / 0022-3700 / 10 / 12 / 010$ 